\title{
InGaN Nanorods Decorated with Au Nanoparticles for Enhanced Water Splitting Based on Surface Plasmon Resonance Effects
}

\author{
Qing Liu ${ }^{1}$, Jiang Shi ${ }^{1}$, Zhenzhu Xu ${ }^{2}$, Bolin Zhang ${ }^{1}$, Hongliang Liu ${ }^{1}$, Yinlei Lin ${ }^{3}$, \\ Fangliang Gao ${ }^{1, * \mathbb{D}}$, Shuti $\mathrm{Li}^{1}$ and Guoqiang $\mathrm{Li}^{2, *}$ \\ 1 Guangdong Engineering Research Center of Optoelectronic Functional Materials and Devices, Institute of \\ Semiconductors, South China Normal University, Guangzhou 510631, China; \\ 2018022795@m.scnu.edu.cn (Q.L.); 2018022807@m.scnu.edu.cn (J.S.); berlinzhang@foxmail.com (B.Z.); \\ 2019022815@m.scnu.edu.cn (H.L.); lishuti@scnu.edu.cn (S.L.) \\ 2 State Key Laboratory of Luminescent Materials and Devices, South China University of Technology, \\ Guangzhou 510640, China; zhenzhupearl@163.com \\ 3 School of Materials Science and Energy Engineering, Foshan University, Foshan 528000, China; \\ linyinlei@fosu.edu.cn \\ * Correspondence: gaofl@m.scnu.edu.cn (F.G.); msgli@scut.edu.cn (G.L.)
}

Received: 13 April 2020; Accepted: 4 May 2020; Published: 9 May 2020

check for updates

\begin{abstract}
Photoelectrochemical (PEC) water splitting has great application potential in converting solar energy into hydrogen energy. However, what stands in the way of the practical application of this technology is the low conversion efficiency, which can be promoted by optimizing the material structure and device design for surface functionalization. In this work, we deposited gold nanoparticles (Au NPs) with different loading densities on the surface of InGaN nanorod (NR) arrays through a chemical solvent route to obtain a composite PEC water splitting system. Enhanced photocatalytic activity, which can be demonstrated by the surface plasmon resonance (SPR) effect induced by Au NPs, occurred and was further confirmed to be associated with the different loading densities of Au NPs. These discoveries use solar water splitting as a platform and provide ideas for exploring the mechanism of SPR enhancement.
\end{abstract}

Keywords: PEC water splitting; InGaN nanorods; Au nanoparticles; surface plasmon resonance

\section{Introduction}

The efficient capture and conversion of solar energy with semiconductors has drawn tremendous interest, for it generates clean and sustainable hydrogen energy without consuming electricity [1,2]. In this regard, solar water splitting provides a feasible way to achieve this vision [3]. Photoelectrochemical (PEC) water splitting converts solar energy through three basic processes: (1) light absorption and excitation of photogenerated carriers, (2) separation of photogenerated carriers and migration to the semiconductor surface, and (3) redox reactions induced by photogenerated carriers [1]. Since Fujishima and Honda used $\mathrm{TiO}_{2}$ to achieve PEC water splitting in 1972, researchers have made unremitting efforts to develop high-efficiency solar-hydrogen conversion materials [4].

Among numerous semiconductor materials, III-nitride semiconductors have been regarded as promising candidates for PEC water splitting; this originates from their tunable energy bandgaps, which span nearly the entire solar spectrum [5-7]. In addition, the band edge has good energy alignment with the water redox potential, which meets the thermodynamic requirements of water splitting $[1,7,8]$. So far, research based on III-nitride semiconductors has been carried out through various low-dimensional structures. Compared with their bulk and film, low-dimensional structures 
have a larger active area and lower light reflectivity, which can strengthen light absorption $[3,9,10]$. Surface band bending is also beneficial for improving carrier separation efficiency and reducing the recombination probability during migration [11,12]. Furthermore, the transmission distance of photogenerated carriers to the semiconductor-electrolyte interface will be greatly reduced with a lower-dimensional structure [13,14].

Functionalizing semiconductor surfaces with plasmonic nanoparticles (NPs), which might cause surface plasmon resonance (SPR) after irradiation, can further enhance light absorption and carrier density [15-17]. The combination of semiconductors and plasmonic NPs constructs a composite material system in which plasmon resonance of NPs enhances the PEC performance based on four significant mechanisms: (1) hot electron injection (HEI) enhancement induced by SPR, (2) light-scattering (trapping) enhancement, (3) plasmon resonance energy transfer (PRET), and (4) light-concentrating enhancement [18-20]. The essence of HEI enhancement is that NPs are excited when electrons are transferred to the conduction band of the semiconductor. In this mechanism, photons with energy below the semiconductor bandgap but higher than the metal-semiconductor Schottky barrier can also contribute to PEC water splitting [15]. Light-scattering enhancement occurs because NPs will first scatter incident light onto the semiconductor surface, thereby reducing the reflection of light from the semiconductor. This effect is more prominent with NPs that are larger than $100 \mathrm{~nm}[21,22]$. Plasmon resonance energy transfer enhances the photocatalytic activity of semiconductors by enhancing carrier generation [23]. Finally, the interaction of NPs and light leads to an enhancement of local electromagnetic fields on the semiconductor surface, which in turn results in the light concentration effect.

To date, numerous reports on NP-enhanced water splitting have illustrated the interest in this method. For instance, Kang et al. reported an $\mathrm{Au} / \mathrm{ZnO}$ composite that exhibits enhanced solar-to-hydrogen efficiency of $0.52 \%$, compared with that of bare $\mathrm{ZnO}(0.24 \%)$; they revealed that the enhancement is caused by light-scattering and HEI [24]. Similarly, Qian et al. reported that the size of $\mathrm{Au}$ NPs plays an important role in the decoration of $\mathrm{TiO}_{2}$, which is directly related to the strength of the HEI effect [25]. Shin et al. also found that the photocatalytic efficiency of Au NPs that decorated $\mathrm{ZnO}-\mathrm{TiO}_{2}$ was promoted because of $\mathrm{HEI}$, and that the effect is loading density-dependent [26]. Hou reported that the photo-to-current efficiency of the $\mathrm{TiO}_{2} / \mathrm{Au} / \mathrm{InGaN}$ sandwich structure was enhanced, which can be explained by HEI [27]. Claverie et al. observed an HEI effect on $\mathrm{Au} / \mathrm{TiO}_{2}$, and the absorption can be increased by more than 40 times [28]. Liu et al. reported that $\mathrm{Au} / \mathrm{Ni} \mathrm{NPs}$ deposited on a $\mathrm{TiO}_{2} / \mathrm{n}$-Si photoanode have 3-fold efficiency under illumination, which can be ascribed to the PRET effect [29]. Basu et al. revealed that $\mathrm{ZnO}$ nanosheets decorated with Au NPs have evident light-scattering and light-absorbing effects [15]. Sang et al. found that the incident photon-to-electron conversion efficiency of InGaN/GaN multiple-quantum-well nanorod-based photoelectrodes (PEs) reached up to $64 \%$ after coupling with $\mathrm{Ag}-\mathrm{Au}$ core-shell nanowires [30].

From the published literature, it can be inferred that NPs have a great effect on the improvement of photocatalytic activity, and this effect is closely related to the size and loading density of the NPs. In this work, InGaN nanorod (NR) arrays decorated with Au NPs were fabricated. NR arrays were first synthesized through molecular-beam epitaxy (MBE), and Au NPs of different loading densities were then deposited. The results indicate that InGaN NRs decorated with Au NPs have a better PEC activity compared to bare InGaN NRs, and that the performance can be optimized by using different loading densities. This enhancement is mainly attributed to the SPR effect from Au NPs.

\section{Experimental Section}

\subsection{MBE Growth of InGaN NRs on Si (111) Wafers}

The $\mathrm{Si}$ (111) wafers were washed three times with acetone, isopropanol, deionized water, and dried with $\mathrm{N}_{2}$ before preparing the InGaN NRs. A radio-frequency plasma-assisted MBE system (PA-MBE, MANTIS, UK) was used to grow InGaN NRs on bare Si wafers. The growth conditions of InGaN NRs were fixed on an in-beam equivalent pressure (BEP) of $4 \times 10^{-7}$ Torr, Ga BEP of $7.2 \times 10^{-8}$ Torr, 
a forward plasma power of $400 \mathrm{~W}$, and a $\mathrm{N}_{2}$ flux of $2.0 \mathrm{sccm}$. The temperature and time required for growth were $900{ }^{\circ} \mathrm{C}$ and $3 \mathrm{~h}$, respectively, as described previously [31-35].

\subsection{Decoration of $A u$ NPs on InGaN NRs}

$\mathrm{Au}$ NPs were deposited on InGaN NRs with ionic layer adsorption and thermal-reduction methods. First, InGaN NRs were immersed into the $\mathrm{HAuCl}_{4}$ aqueous solution for $12 \mathrm{~h}$ in the dark and then blown dry with $\mathrm{N}_{2}$. Au NPs with different particle sizes and growth densities were obtained by using different concentrations of $\mathrm{HAuCl} 4$ aqueous solution. The concentrations of the $\mathrm{HAuCl}_{4}$ aqueous solutions were 5, 10, and $15 \mu \mathrm{M}$ and were marked as Au5@NRs, Au10@NRs, and Au15@NRs, respectively. A mixed solution of $4 \mathrm{~mL}$ of absolute ethanol and $6 \mathrm{~mL}$ of deionized water was heated using a device (HWJB-2100CT, Zhengzhou Carbon State Instrument Co., Ltd., China) set to $110{ }^{\circ} \mathrm{C}$. The as-grown wafer was immersed in the mixed solution for $10 \mathrm{~min}$. Finally, the as-grown wafer was washed with deionized water and dried with $\mathrm{N}_{2}$.

\subsection{Materials Characterization}

The morphology of InGaN NRs was characterized by scanning electron microscopy (SEM, ZEISS Ultra 55, Oberkochen, Germany) and transmission electron microscopy (TEM JEOL 3000F, FEI, Japan). The UV-vis absorption spectrum of the sample was characterized using an UV-vis spectrometer (Cary 500, Varian, Palo Alto, CA, USA).

\subsection{PEC Measurements}

PEC measurements were carried out on a three-electrode electrochemical workstation $(\mathrm{CHI} 760 \mathrm{E}$, $\mathrm{CH}$ Instruments Inc., Austin, TX, USA) In the PEC system of this experiment, the as-prepared sample was the working electrode, the platinum wire was the counter electrode, the saturated calomel electrode (saturated $\mathrm{KCl}, \mathrm{SCE}$ ) was the reference electrode, and the electrolyte was a $0.5 \mathrm{M} \mathrm{H}_{2} \mathrm{SO}_{4}(\mathrm{pH}=0)$ solution. A $300 \mathrm{~W}$ Xe lamp $\left(100 \mathrm{~mW} \mathrm{~cm}^{-2}\right)$ was used to illuminate the sample. The working area of the photoanode was $0.3-0.6 \mathrm{~cm}^{2}$. Unless otherwise specified, all potentials in this experiment were reported with respect to SCE.

\section{Results and Discussion}

The design and fabrication process of decorating Au NPs on InGaN NR arrays is shown in Figure 1. It can be found from the figure that the complete experiment is mainly divided into two parts. First, MBE was used to grow an InGaN NR array on a Si substrate, and then the NRs were decorated with $\mathrm{Au}$ NPs using ion exchange. The detailed growth process and steps can be seen in the experimental section.

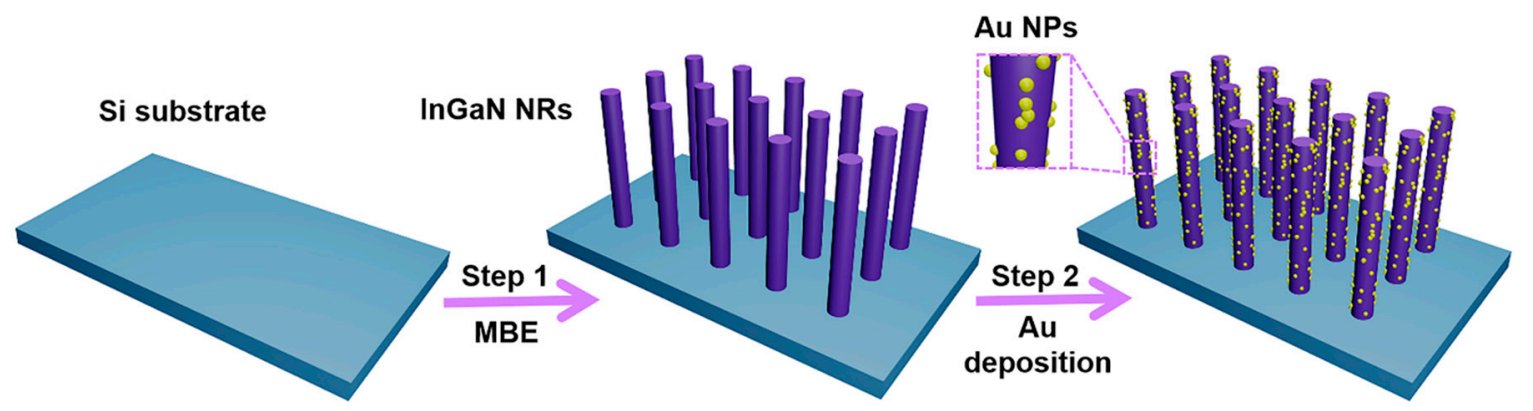

Figure 1. Schematic diagram of the preparation process for decoration of Au nanoparticles (NPs) on InGaN nanorod (NR) arrays.

The surface morphology of semiconductor materials is very important for the PEC water splitting system. From Figure 2a, it can be obtained that the prepared InGaN NRs have high density. The surface of the InGaN NRs is very smooth, which indicates the high quality of the as-grown InGaN NRs. The Au 
NPs decorated on the surfaces of the InGaN NRs had different loading densities, and the corresponding SEM images are shown in Figure $2 b-d$. From the figure, it can be seen that the SEM images of the deposited Au NPs have a clear graininess compared to those of the undeposited, and the graininess becomes stronger as the concentration of the $\mathrm{HAuCl}_{4}$ aqueous solution increases. This is mainly attributed to the fact that more Au NPs are deposited on the nanopillars under the same conditions through the ion exchange reaction. However, one cannot determine from the SEM images whether Au NPs are deposited on the InGaN NRs, and the size of Au NPs cannot be observed. Therefore, TEM was used for further characterization. Figure 2e, f shows the High-resolution TEM (HRTEM) diagram of a single Au@NR, in which it can be clearly seen that there is good contact between the Au NPs and the InGaN NRs. Figure $2 \mathrm{f}$ shows the well-resolved highly crystalline structure along the (0001) plane of InGaN NRs $(\mathrm{d}=0.536 \mathrm{~nm})$, as well as along the (111) plane of the face-centered cubic Au $(\mathrm{d}=0.24 \mathrm{~nm})$, and the diameter of the Au NPs is $\sim 5 \mathrm{~nm}$. For samples Au10@NR and Au15@NR, their particle sizes are 11 and $16 \mathrm{~nm}$, respectively. An energy dispersive spectrometer (EDS, ZEISS Ultra 55, Oberkochen, Germany) was used for verification of the densities of the Au NPs. Figure S1 shows the EDS diagram of InGaN NRs with $\mathrm{Au}$ NPs deposited using $\mathrm{HAuCl}_{4}$ aqueous solutions of different concentrations. EDS analysis indicates that the sample has a high Au content, and the Au content increases as the concentration of the immersion solution increases. This is consistent with the previous inference.
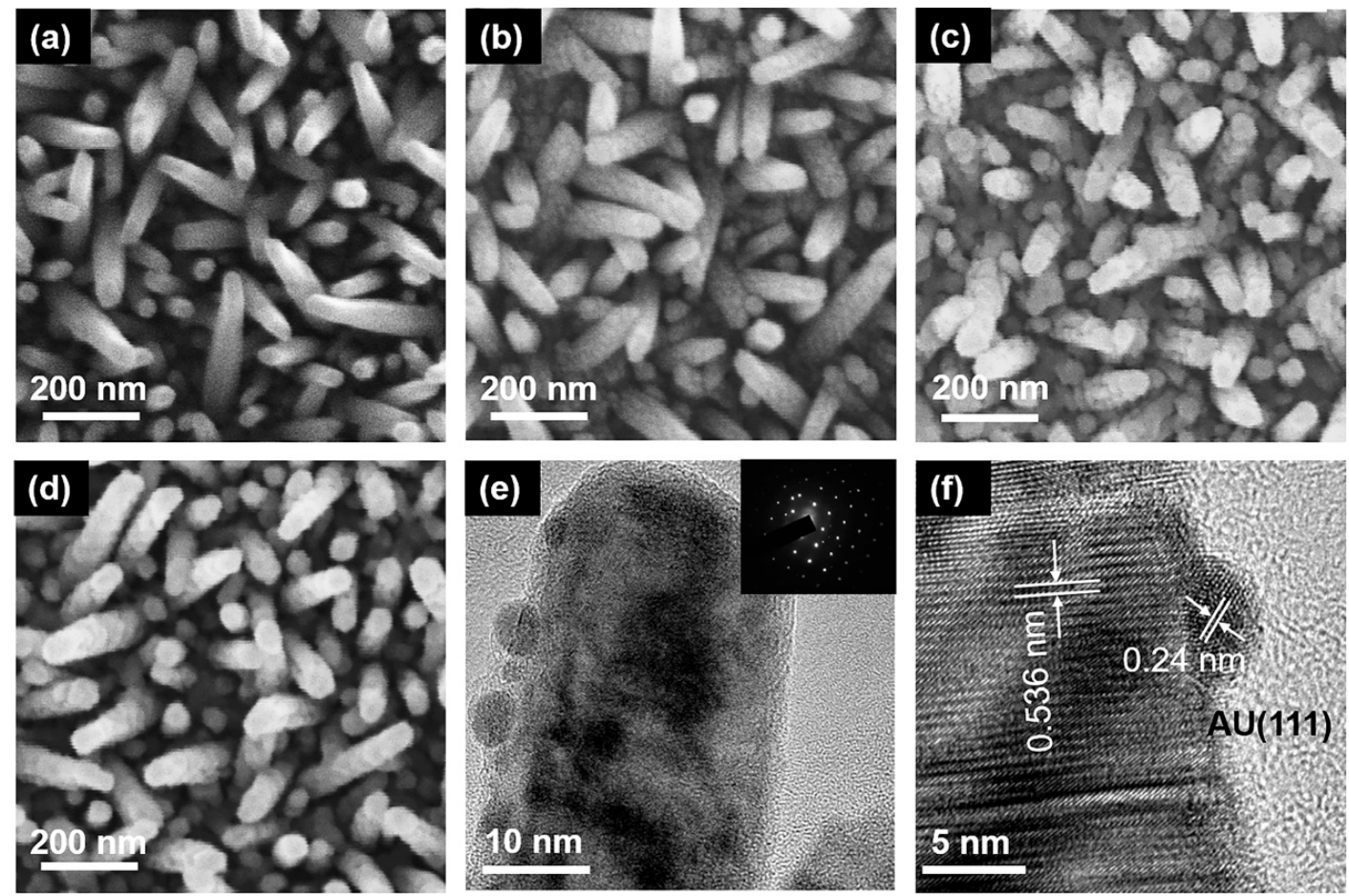

Figure 2. SEM images of InGaN (a) bare NRs, (b) Au5@NRs, (c) Au10@NRs, and (d) Au15@NRs. (e) TEM image of $\mathrm{Au} @ \mathrm{NR}$, inset is the corresponding selected area electron diffraction. (f) High-resolution TEM image of Au5@NR.

The light absorption characteristic of the InGaN NRs photoelectrode is very important for the efficiency of PEC water splitting. Figure 3 shows the absorption spectra of bare NRs, Au5@NRs, Au10@NRs, and Au15@NRs. It can be seen from the figure that InGaN has an absorption center at $460 \mathrm{~nm}$, and an absorption cut-off peak around $650 \mathrm{~nm}$. This absorption spectrum of InGaN NRs is mainly caused by the direct band-to-band transition [31]. However, after the Au NPs are deposited on the InGaN NRs, the intensity of the absorption peak decreases as the deposition density increases. After the NPs are deposited, the absorption of light on the surface of the InGaN NRs is hindered, which affects the direct band-to-band transition and weakens the absorption peak. In addition, the broad 
long-wavelength absorption is attributed to a spatially indirect transition [31]. Thus, InGaN NRs have better light absorption in the near-infrared short-wave region (780-1100 nm), whereas there is almost no significant absorption in the subsequent regions, which can be clearly distinguished in Figure 3. However, when Au NPs are used to decorate the InGaN NRs, not only is the light absorption of the InGaN NRs in the near-infrared short-wave region increased, but also the absorption region is extended to the near-infrared long-wave region. Enhanced light absorption may be attributed to SPR excitation of Au NPs [24,36-40], and the shift of the long-wavelength absorption edge (1000 $\mathrm{nm})$ is due to the intrinsic absorption characteristics of Au NPs [19]. Interestingly, the sample also shows an absorption peak around $850 \mathrm{~nm}$, which is mainly the absorption peak of InGaN [41]. From Figure 3, it also can be clearly seen that the absorption spectra of different growth concentrations have different absorption intensities. A higher absorption intensity was observed when fabricating the photoelectrode using higher concentration $\mathrm{HAuCl}_{4}$ aqueous solutions. This can be ascribed to the higher density of Au NPs decorating the surface of the InGaN NRs, and the larger particle size at higher concentrations with the same conditions [40]. This result is in good agreement with the previous SEM and EDS results.

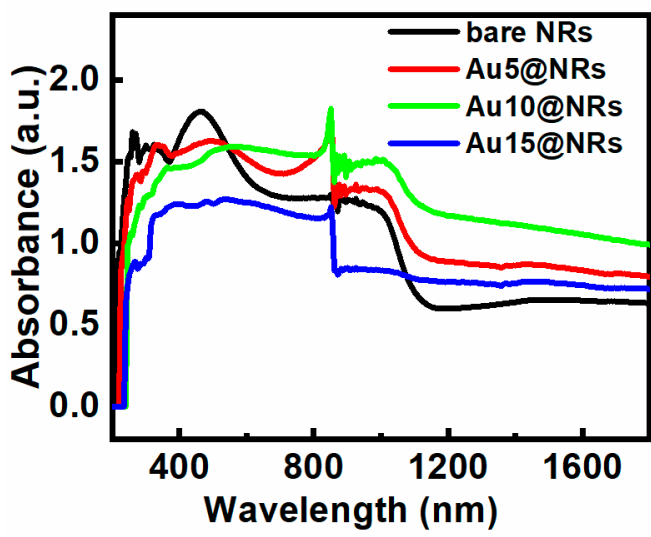

Figure 3. Absorption spectra of bare NRs, Au5@NRs, Au10@NRs, and Au15@NRs.

To study the effect of deposition of Au NPs on the InGaN NRs photoelectrode, InGaN NRs with different densities of $\mathrm{Au}$ NPs deposited onto them were prepared. Figure $4 \mathrm{a}$ reflects the linear scan curves of different samples under light and dark conditions. From Figure $4 \mathrm{a}$, it can be found that after depositing Au NPs, the photocurrent density of the photoelectrode increases remarkably with the increase of Au NP deposition density. The dark current of the photoelectrode is hardly changed with the deposition of Au NPs, which indicates that the sample has a high crystal quality. However, when the current density of the Au10@NRs reaches its highest value, further improvement of the concentration of $\mathrm{HAuCl}_{4}$ aqueous solutions is harmful for device performance, which manifests as a decrease in the photocurrent density as the concentration of $\mathrm{HAuCl}_{4}$ aqueous solutions increases. This can be explained by using a higher concentration of $\mathrm{HAuCl}_{4}$ aqueous solutions to prepare samples under the same conditions so that the surface of the InGaN NRs is mostly covered by a large amount of Au NPs, which blocks the surface of the InGaN NRs from absorbing light, thereby reducing the generation of photogenerated electron-hole pairs. The high coverage of the Au NPs blocks the contact of the surface of the InGaN NRs with the electrolyte and hinders the water oxidation reaction [26,42]. This shows that the deposition of Au NPs with an appropriate density has a significant improvement in the efficiency of the InGaN NRs PEC water splitting system. In addition, the enhanced photocurrent density can be ascribed to several effects. Firstly, Au NPs increase light absorption by the SPR effect. Secondly, Au NPs capture photogenerated electrons from InGaN NRs for water reduction and reduce the recombination of photogenerated electrons and holes [43,44]. Thirdly, Au NPs generate hot plasmon to excite electrons to directly affect water reduction [45]. Finally, Au NPs form a Schottky contact with the InGaN NR surface, thereby forming a built-in electric field to promote the separation of photogenerated electrons and holes. Figure $4 \mathrm{~b}$ shows the I-t curves of the bare NRs, Au5@NRs, 
Au10@NRs, and Au15@NRs under chopped illumination at 0.6 V. All samples showed extremely low current density in the dark. Photocurrent density rapidly increases to its highest value under light conditions. The photocurrent density and on/off ratio of the sample deposited with $10 \mu \mathrm{M} \mathrm{HACl}_{4}$ aqueous solution are the largest. The sharp increase in photocurrent density from off to on states indicates that the sample has a significant photoresponse. There is effective generation and separation of photogenerated electrons and holes, and the rapid transport of electrons from the Au NPs to the InGaN NR surface, which promotes the progress of the water splitting. In addition, the photocurrent density of the samples is only slightly reduced after $400 \mathrm{~s}$, which indicates that the samples have extremely strong stability. In summary, samples made with $10 \mu \mathrm{M} \mathrm{HAuCl}_{4}$ aqueous solution show the best PEC water splitting performance.
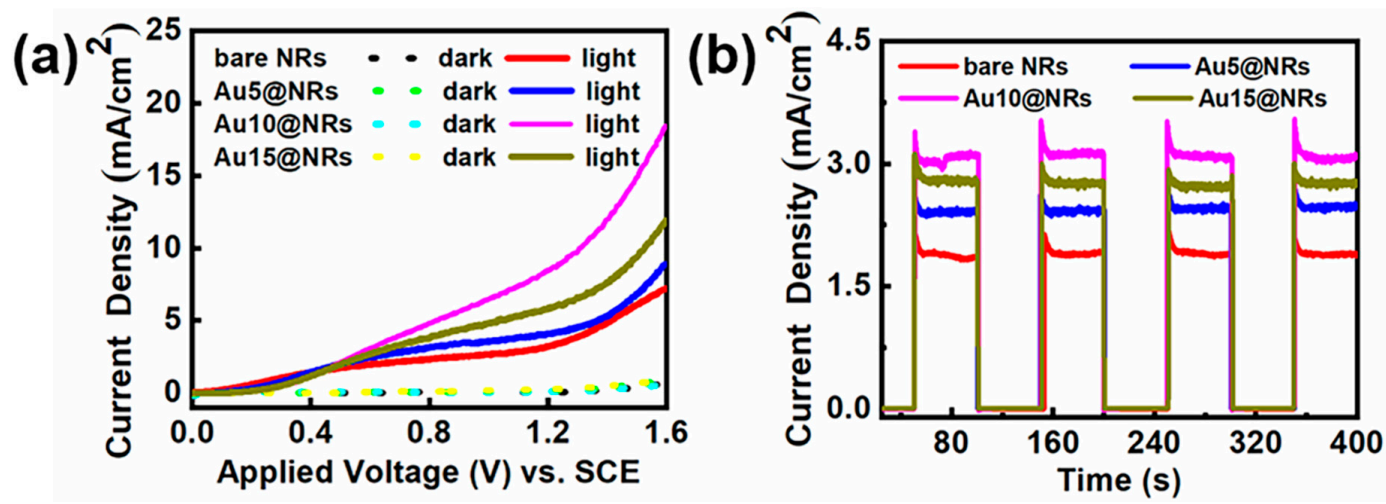

Figure 4. (a) Current-potential curves of the bare NRs, Au5@NRs, Au10@NRs, and Au15@NRs photoanodes in $0.5 \mathrm{M} \mathrm{H}_{2} \mathrm{SO}_{4}$ solution in the dark and under $100 \mathrm{~mW} \mathrm{~cm}{ }^{-2}$ irradiation. (b) The amperometric I-t curves of the bare NRs, Au5@NRs, Au10@NRs, and Au15@NRs under chopped illumination at $0.6 \mathrm{~V}$ vs. reversible hydrogen electrode (RHE).

To further study the enhancement mechanism of the InGaN Au10@NRs, a series of electrochemical measurements was performed and is shown in Figure 5. Figure 5a shows the Nyquist diagram of the sample. This is the use of electrochemical impedance spectra to evaluate the carrier transfer process of the sample, and the radius of the Nyquist plot circle represents the charge transfer resistance. Au10@NRs show smaller charge transfer resistance compared to bare NRs, which indicates that Au NPs can increase the electric conductivity of InGaN NRs, thereby increasing the carrier transport speed between the Au NPs and InGaN NRs interface. Photoelectrode stability is critical for the PEC water splitting system, and Figure $5 \mathrm{~b}$ shows the photocurrent density-time curve of bare InGaN NRs and InGaN Au10@NRs with an applied bias of $0.6 \mathrm{~V}$ in $0.5 \mathrm{M} \mathrm{H}_{2} \mathrm{SO}_{4}$ solution. It is clearly shown that the photocurrent densities of bare InGaN NRs and Au10@NRs have only slightly attenuated after a one-hour test, which indicates that the sample has good stability.

(a)

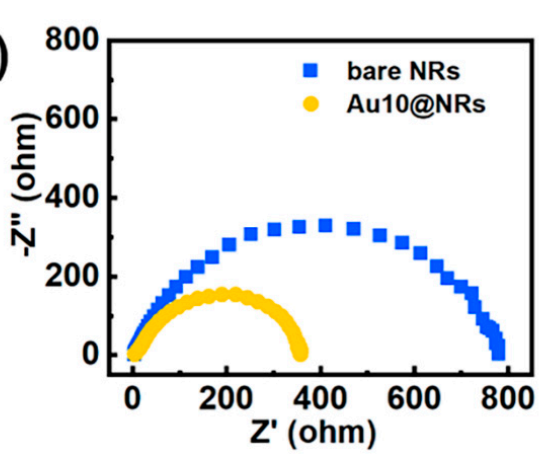

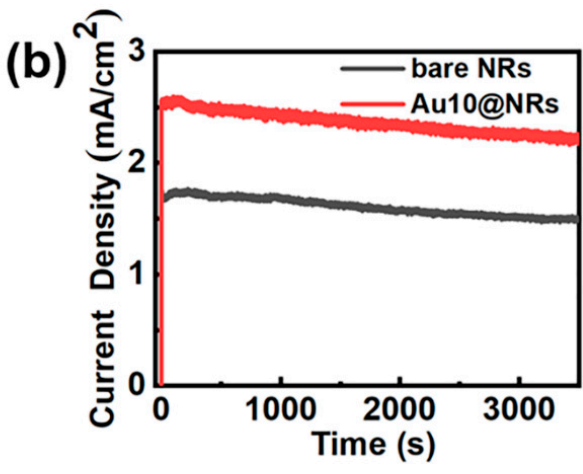

Figure 5. (a) Nyquist plots of bare NRs and Au10@NRs. (b) Stability of the bare NRs and Au10@NRs photoanodes for photoelectrochemical (PEC) the oxygen evolution reaction (OER). 
In order to better understand the carrier transport mechanism, the schematic of mechanisms for the enhanced PEC water splitting efficiency of the InGaN NRs@Au photoelectrode is shown in Figure 6. When the photoanode is irradiated under light, the InGaN NRs are excited to generate photogenerated electron-hole pairs. The photogenerated electrons are transferred to the Si substrate through the InGaN NRs, and then to the Pt electrode through an external circuit. Simultaneously, $\mathrm{Au}$ NPs on the surface of the InGaN NRs absorb light to generate photogenerated electrons due to the SPR effect. The generated hot electrons are injected into the InGaN conduction band, and electrons accumulate in the InGaN conduction band because of the effect of the Schottky barrier, such that more electrons are transported between InGaN and Si and to the cathode through an external circuit. The synergistic effect of Au NPs reduces the conduction band of InGaN and increases light absorption efficiency. Moreover, due to the Schottky contact between Au and InGaN, a built-in electric field is generated to promote the separation of photogenerated electrons and holes and increase the lifetimes of carriers. More electrons are transferred to the Pt electrode to increase the efficiency of the PEC water splitting system. Therefore, the deposition of Au NPs on the surface of InGaN NRs is a feasible method to improve the efficiency of PEC InGaN water splitting.

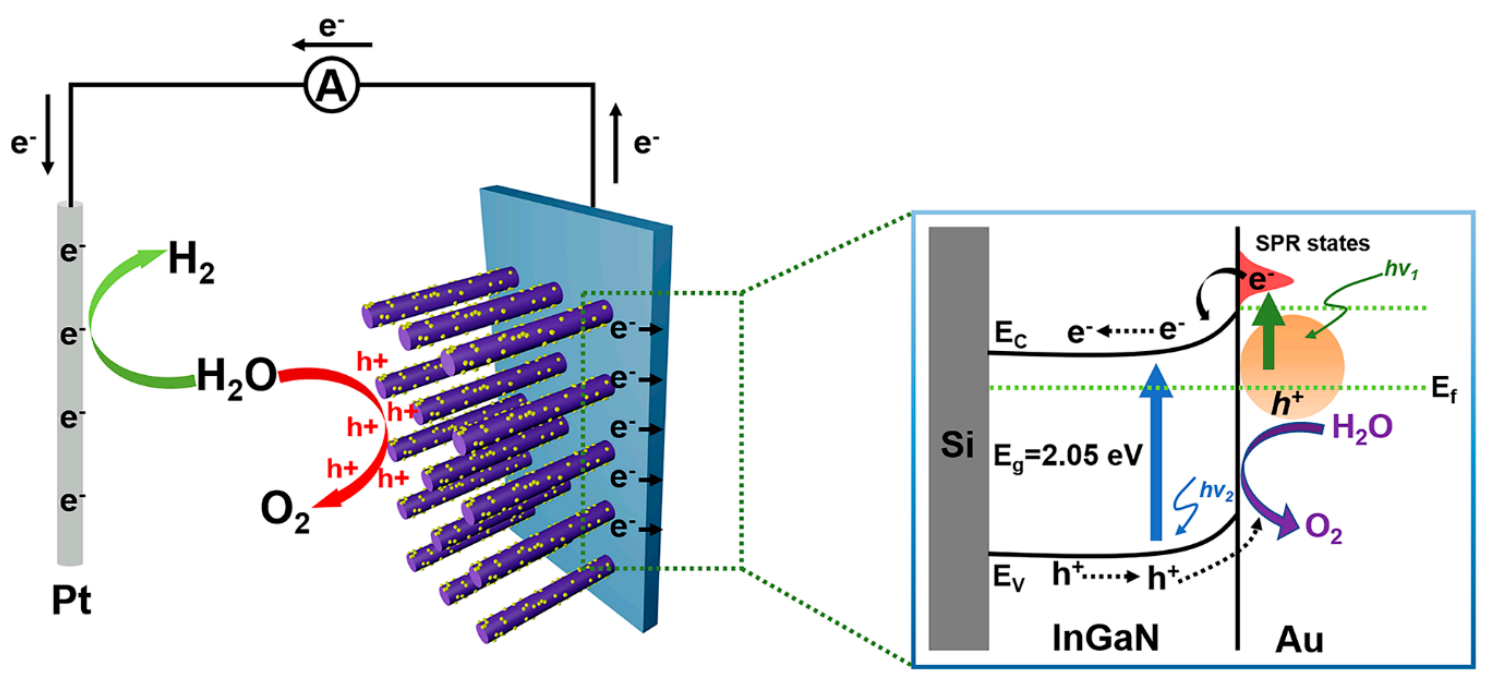

Figure 6. Schematic of mechanisms for understanding the enhanced PEC water splitting efficiency of the Au@InGaN NR photoelectrode.

\section{Conclusions}

We have successfully prepared the Au NPs@InGaN NRs photoelectrode and controlled the growth density of $\mathrm{Au}$ NPs by changing the concentration of the $\mathrm{HAuCl}_{4}$ aqueous solution. It can be found that the deposition of Au NPs on the surface of InGaN NRs can significantly improve the efficiency of water splitting. Moreover, the density of $\mathrm{Au}$ NPs deposited with $10 \mu \mathrm{M} \mathrm{HAuCl}_{4}$ aqueous solutions is the most suitable. The increasing of photocurrent density is mainly attributed to the SPR effect, and it promotes the separation of photogenerated electron-hole pairs. In addition, the NRs structure of InGaN has a larger body surface area to capture more light, and the directional transfer of the NRs promotes the carrier separation and transfer. In conclusion, this work provides a feasible method for improving the efficiency of semiconductor PEC water splitting and has a promoting effect on the practical application of PEC water splitting.

Supplementary Materials: The following are available online at http://www.mdpi.com/2079-4991/10/5/912/s1, Figure S1. EDS images of Au5@NRs, Au10@NRs, and Au15@NRs.

Author Contributions: Conceptualization, Q.L., Z.X. and F.G.; Formal analysis, Q.L., J.S., Z.X., B.Z., H.L. and Y.L.; Writing-original draft, Q.L. and J.S.; Writing—review and editing, Funding acquisition, S.L. and G.L. All authors have read and agreed to the published version of the manuscript.

Funding: This research received no external funding. 
Acknowledgments: This work was supported by the Natural Science Foundation of Guangdong Province, China (2018A030313395), Joint Fund of Ministry of Education for Equipment Pre-Research (6141A02022435), Key Innovation Project in Industry Chain of Shaanxi Province (2018ZDCXL-GY-01-02-01), National Natural Science Foundation of China (51803028), the scientific research start-up fund for high-level talents of Foshan University, China (gg07077), Foshan Functional Polymer Engineering Center (2016GA10162), and the key Project of Department of Education of Guangdong Province (2016GCZX008).

Conflicts of Interest: The authors declare no conflict of interest.

\section{References}

1. Wang, D.; Pierre, A.; Kibria, M.G.; Cui, K.; Han, X.; Bevan, K.H.; Guo, H.; Paradis, S.; Hakima, A.R.; Mi, Z. Wafer-level photocatalytic water splitting on GaN nanowire arrays grown by molecular beam epitaxy. Nano Lett. 2011, 11, 2353-2357. [CrossRef] [PubMed]

2. Zou, Z.; Ye, J.; Sayama, K.; Arakawa, H. Direct splitting of water under visible light irradiation with an oxide semiconductor photocatalyst. Nature 2001, 414, 625-627. [CrossRef] [PubMed]

3. Li, X.; Liu, S.; Fan, K.; Liu, Z.; Song, B.; Yu, J. MOF-Based Transparent Passivation Layer Modified ZnO Nanorod Arrays for Enhanced Photo-Electrochemical Water Splitting. Adv. Energy Mater. 2018, 8, 1800101. [CrossRef]

4. Fujishima, A.; Honda, K. Electrochemical Photolysis of Water at a Semiconductor Electrode. Nature 1972, 238, 37-38. [CrossRef]

5. Fan, S.; AlOtaibi, B.; Woo, S.Y.; Wang, Y.; Botton, G.A.; Mi, Z. High efficiency solar-to-hydrogen conversion on a monolithically integrated InGaN/GaN/Si adaptive tunnel junction photocathode. Nano Lett. 2015, 15, 2721-2726. [CrossRef]

6. Varadhan, P.; Fu, H.C.; Priante, D.; Retamal, J.R.; Zhao, C.; Ebaid, M.; Ng, T.K.; Ajia, I.; Mitra, S.; Roqan, I.S.; et al. Surface Passivation of GaN Nanowires for Enhanced Photoelectrochemical Water-Splitting. Nano Lett. 2017, 17, 1520-1528. [CrossRef]

7. Kim, Y.J.; Lee, G.J.; Kim, S.; Min, J.W.; Jeong, S.Y.; Yoo, Y.J.; Lee, S.; Song, Y.M. Efficient Light Absorption by GaN Truncated Nanocones for High Performance Water Splitting Applications. ACS Appl. Mater. Interfaces 2018, 10, 28672-28678. [CrossRef]

8. Yang, C.; Xi, X.; Yu, Z.; Cao, H.; Li, J.; Lin, S.; Ma, Z.; Zhao, L. Light Modulation and Water Splitting Enhancement Using a Composite Porous GaN Structure. ACS Appl. Mater. Interfaces 2018, 10, 5492-5497. [CrossRef]

9. Pan, K.; Dong, Y.; Zhou, W.; Pan, Q.; Xie, Y.; Xie, T.; Tian, G.; Wang, G. Facile fabrication of hierarchical TiO nanobelt/ZnO nanorod heterogeneous nanostructure: An efficient photoanode for water splitting. ACS Appl. Mater. Interfaces 2013, 5, 8314-8320. [CrossRef]

10. Han, H.; Kment, S.; Karlicky, F.; Wang, L.; Naldoni, A.; Schmuki, P.; Zboril, R. Sb-Doped SnO $2 \mathrm{Nanorods}^{-}$ Underlayer Effect to the $\alpha-\mathrm{Fe}_{2} \mathrm{O}_{3}$ Nanorods Sheathed with $\mathrm{TiO}_{2}$ for Enhanced Photoelectrochemical Water Splitting. Small 2018, 14, e1703860. [CrossRef]

11. Wang, H.; Tian, J.; Li, W. Electrochemical Deposition of MgO@ZnO Shell-Core Nanorod Arrays Largely Enhances the Photoelectrochemical Water Splitting Performance. ChemElectroChem 2017, 4, 2019-2026. [CrossRef]

12. Sun, B.; Chen, Y.; Tao, L.; Zhao, H.; Zhou, G.; Xia, Y.; Wang, H.; Zhao, Y. Nanorod Array of $\mathrm{SnO}_{2}$ Quantum Dot Interspersed Multiphase $\mathrm{TiO}_{2}$ Heterojunctions with Highly Photocatalytic Water Splitting and Self-Rechargeable Battery-Like Applications. ACS Appl. Mater. Interfaces 2019, 11, 2071-2081. [CrossRef] [PubMed]

13. Liao, A.; He, H.; Tang, L.; Li, Y.; Zhang, J.; Chen, J.; Chen, L.; Zhang, C.; Zhou, Y.; Zou, Z. Quasi-Topotactic Transformation of FeOOH Nanorods to Robust $\mathrm{Fe}_{2} \mathrm{O}_{3}$ Porous Nanopillars Triggered with a Facile Rapid Dehydration Strategy for Efficient Photoelectrochemical Water Splitting. ACS Appl. Mater. Interfaces 2018, 10, 10141-10146. [CrossRef] [PubMed]

14. Wang, W.; Jin, C.; Qi, L. Hierarchical CdS Nanorod@SnO $\mathrm{O}_{2}$ Nanobowl Arrays for Efficient and Stable Photoelectrochemical Hydrogen Generation. Small 2018, 14, e1801352. [CrossRef] [PubMed]

15. Mahala, C.; Sharma, M.D.; Basu, M. Near-Field and Far-Field Plasmonic Effects of Gold Nanoparticles Decorated on ZnO Nanosheets for Enhanced Solar Water Splitting. ACS Appl. Nano Mater. 2020, 3, 1153-1165. [CrossRef] 
16. Zhou, X.; Liu, G.; Yu, J.; Fan, W. Surface plasmon resonance-mediated photocatalysis by noble metal-based composites under visible light. J. Mater. Chem. 2012, 22, 21337-21354. [CrossRef]

17. Zhang, H.; Chen, G.; Bahnemann, D.W. Photoelectrocatalytic materials for environmental applications. J. Mater. Chem. 2009, 19, 5089-5121. [CrossRef]

18. Liu, Z.; Hou, W.; Pavaskar, P.; Aykol, M.; Cronin, S.B. Plasmon resonant enhancement of photocatalytic water splitting under visible illumination. Nano Lett. 2011, 11, 1111-1116. [CrossRef]

19. Hu, D.; Diao, P.; $\mathrm{Xu}, \mathrm{D} . ; \mathrm{Wu}, \mathrm{Q}$. Gold $/ \mathrm{WO}_{3}$ nanocomposite photoanodes for plasmonic solar water splitting. Nano Res. 2016, 9, 1735-1751. [CrossRef]

20. Knight, M.W.; Sobhani, H.; Nordlander, P.; Halas, N.J. Photodetection with active optical antennas. Science 2011, 332, 702-704. [CrossRef]

21. Warren, S.C.; Thimsen, E. Plasmonic solar water splitting. Energy Environ. Sci. 2012, 5, 5133-5146. [CrossRef]

22. Erwin, W.R.; Zarick, H.F.; Talbert, E.M.; Bardhan, R. Light trapping in mesoporous solar cells with plasmonic nanostructures. Energy Environ. Sci. 2016, 9, 1577-1601. [CrossRef]

23. Thimsen, E.; Le Formal, F.; Gratzel, M.; Warren, S.C. Influence of plasmonic Au nanoparticles on the photoactivity of $\mathrm{Fe}_{2} \mathrm{O}_{3}$ electrodes for water splitting. Nano Lett. 2011, 11, 35-43. [CrossRef] [PubMed]

24. Zhang, X.; Liu, Y.; Kang, Z. 3D branched ZnO nanowire arrays decorated with plasmonic Au nanoparticles for high-performance photoelectrochemical water splitting. ACS Appl. Mater. Interfaces 2014, 6, 4480-4489. [CrossRef]

25. Qian, K.; Sweeny, B.C.; Johnston-Peck, A.C.; Niu, W.; Graham, J.O.; DuChene, J.S.; Qiu, J.; Wang, Y.C.; Engelhard, M.H.; Su, D.; et al. Surface plasmon-driven water reduction: Gold nanoparticle size matters. J. Am. Chem. Soc. 2014, 136, 9842-9845. [CrossRef] [PubMed]

26. Park, J.; Deshmukh, P.R.; Sohn, Y.; Shin, W.G. ZnO-TiO 2 core-shell nanowires decorated with Au nanoparticles for plasmon-enhanced photoelectrochemical water splitting. J. Alloys Compd. 2019, 787, 1310-1319. [CrossRef]

27. Hou, Y. Engineering hot electrons of localized surface plasmon on InGaN photoanode for solar-powered water splitting. J. Photonics Energy 2019, 9, 026001. [CrossRef]

28. Zhang, J.; Jin, X.; Morales-Guzman, P.I.; Yu, X.; Liu, H.; Zhang, H.; Razzari, L.; Claverie, J.P. Engineering the Absorption and Field Enhancement Properties of $\mathrm{Au}-\mathrm{TiO}_{2}$ Nanohybrids via Whispering Gallery Mode Resonances for Photocatalytic Water Splitting. ACS Nano 2016, 10, 4496-4503. [CrossRef]

29. Hong, W.; Cai, Q.; Ban, R.; He, X.; Jian, C.; Li, J.; Li, J.; Liu, W. High-Performance Silicon Photoanode Enhanced by Gold Nanoparticles for Efficient Water Oxidation. ACS Appl. Mater. Interfaces 2018, 10, 6262-6268. [CrossRef]

30. Sang, Y.; Liu, B.; Tao, T.; Jiang, D.; Wu, Y.; Chen, X.; Luo, W.; Ye, J.; Zhang, R. Plasmon-enhanced photoelectrochemical water splitting by InGaN/GaN nano-photoanodes. Semicond. Sci. Technol. 2020, 35, 025017. [CrossRef]

31. Xu, Z.; Zhang, S.; Gao, F.; Wen, L.; Yu, Y.; Li, G. Correlations among morphology, composition, and photoelectrochemical water splitting properties of InGaN nanorods grown by molecular beam epitaxy. Nanotechnology 2018, 29, 475603. [CrossRef] [PubMed]

32. Xu, Z.; Zhang, S.; Liang, J.; Lin, J.; Yu, Y.; Li, R.; Gao, F.; Li, G. Surface passivation of InGaN nanorods using $\mathrm{H}_{3} \mathrm{PO}_{4}$ treatment for enhanced photoelectrochemical performance. J. Power Sources 2019, 419, 65-71. [CrossRef]

33. Lin, J.; Yu, Y.; Zhang, Z.; Gao, F.; Liu, S.; Wang, W.; Li, G. A Novel Approach for Achieving High-Efficiency Photoelectrochemical Water Oxidation in InGaN Nanorods Grown on Si System: MXene Nanosheets as Multifunctional Interfacial Modifier. Adv. Funct. Mater. 2020, 30, 1910479. [CrossRef]

34. Xu, Z.; Zhang, S.; Gao, F.; Gao, P.; Yu, Y.; Lin, J.; Liang, J.; Li, G. Enhanced charge separation and interfacial charge transfer of InGaN nanorods/ $\mathrm{C}_{3} \mathrm{~N}_{4}$ heterojunction photoanode. Electrochim. Acta 2019, 324, 134844. [CrossRef]

35. Lin, J.; Yu, Y.; Xu, Z.; Gao, F.; Zhang, Z.; Zeng, F.; Wang, W.; Li, G. Electronic engineering of transition metal Zn-doped InGaN nanorods arrays for photoelectrochemical water splitting. J. Power Sources 2020, 450, 227578. [CrossRef]

36. Lu, Y.; Zhang, J.; Ge, L.; Han, C.; Qiu, P.; Fang, S. Synthesis of novel AuPd nanoparticles decorated one-dimensional $\mathrm{ZnO}$ nanorod arrays with enhanced photoelectrochemical water splitting activity. J. Colloid Interface Sci. 2016, 483, 146-153. [CrossRef] 
37. Guo, C.X.; Xie, J.; Yang, H.; Li, C.M. Au@CdS Core-Shell Nanoparticles-Modified ZnO Nanowires Photoanode for Efficient Photoelectrochemical Water Splitting. Adv. Sci. 2015, 2, 1500135. [CrossRef]

38. Liu, Y.; Yan, X.; Kang, Z.; Li, Y.; Shen, Y.; Sun, Y.; Wang, L.; Zhang, Y. Synergistic Effect of Surface Plasmonic particles and Surface Passivation layer on ZnO Nanorods Array for Improved Photoelectrochemical Water Splitting. Sci. Rep. 2016, 6, 29907. [CrossRef]

39. Zhang, Z.; Zhang, L.; Hedhili, M.N.; Zhang, H.; Wang, P. Plasmonic gold nanocrystals coupled with photonic crystal seamlessly on $\mathrm{TiO}_{2}$ nanotube photoelectrodes for efficient visible light photoelectrochemical water splitting. Nano Lett. 2013, 13, 14-20. [CrossRef]

40. Linic, S.; Christopher, P.; Ingram, D.B. Plasmonic-metal nanostructures for efficient conversion of solar to chemical energy. Nat. Mater. 2011, 10,911-921. [CrossRef]

41. Mauder, C.; Tuna, Ö.; Gutrath, B.; Balmes, V.; Behmenburg, H.; Rzheutskii, M.V.; Lutsenko, E.V.; Yablonskii, G.P.; Noyong, M.; Simon, U.; et al. Highly n-type doped InGaN films for efficient direct solar hydrogen generation. Phys. Status Solidi C 2012, 9, 964-967. [CrossRef]

42. Chen, H.M.; Chen, C.K.; Chen, C.J.; Cheng, L.C.; Wu, P.C.; Cheng, B.H.; Ho, Y.Z.; Tseng, M.L.; Hsu, Y.Y.; Chan, T.S.; et al. Plasmon inducing effects for enhanced photoelectrochemical water splitting: $\mathrm{X}$-ray absorption approach to electronic structures. ACS Nano 2012, 6, 7362-7372. [CrossRef] [PubMed]

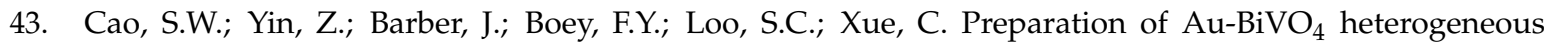
nanostructures as highly efficient visible-light photocatalysts. ACS Appl. Mater. Interfaces 2012, 4, 418-423. [CrossRef] [PubMed]

44. Subramanian, V.; Wolf, E.E.; Kamat, P.V. Catalysis with $\mathrm{TiO}_{2} /$ gold nanocomposites. Effect of metal particle size on the Fermi level equilibration. J. Am. Chem. Soc. 2004, 126, 4943-4950. [CrossRef] [PubMed]

45. Chang, S.; Li, Q.; Xiao, X.; Wong, K.Y.; Chen, T. Enhancement of low energy sunlight harvesting in dye-sensitized solar cells using plasmonic gold nanorods. Energy Environ. Sci. 2012, 5, 9444-9448. [CrossRef]

(C) 2020 by the authors. Licensee MDPI, Basel, Switzerland. This article is an open access article distributed under the terms and conditions of the Creative Commons Attribution (CC BY) license (http://creativecommons.org/licenses/by/4.0/). 